\title{
A Rare Case of Cushing's Syndrome caused by 'cyclical' Ectopic ACTH secretion
}

\author{
Behary $\mathrm{P}^{1}$, Kadiyala $\mathbf{R}^{1}$, Falinska $\mathrm{A}^{1}$, Beckwith $\mathrm{H}^{1}$, Jackson $\mathrm{J}^{1}$, Mitchell $\mathrm{C}^{2}$, Todd $\mathrm{JF}^{1}$ \\ 1 Imperial Centre for Endocrinology, Imperial college Healthcare NHS Trust, Hammersmith Hospital, Du Cane road, London UK \\ 2 Diabetes and Endocrinology, The Hillingdon Hospital NHS Trust
}

\section{Case Report}

A 61 year old lady presented in April 2013 with rapid onset of lethargy and reduced mobility with inability to use stairs over one month. Prior to this, she was fit and well and had no significant medical history and was a lifelong non-smoker. At presentation, it was noted that she was overweight with a BMI of $35 \mathrm{~kg} / \mathrm{m} 2$ and had evidence of skin bruising, severe proximal myopathy of her legs and bilateral leg cellulitis.

\section{Investigations}

A midnight cortisol was $>1710 \mathrm{nmol} / \mathrm{l}$ with a corresponding ACTH of $610 \mathrm{nmol} / \mathrm{L}$ confirming ACTH dependent Cushing's syndrome. Serum potassium was $2.6 \mathrm{mmol} / \mathrm{l}$ and a new diagnosis of type 2 diabetes was made. Her inpatient stay was complicated by bilateral pneumonia requiring non-invasive ventilation. She was commenced on metyrapone $500 \mathrm{mg}$ tds for her significant disease burden. MRI of her pituitary revealed no lesion and CT scan of adrenals showed bilateral adrenal hyperplasia consistent with ACTH dependent Cushing's syndrome. In preparation for IPSS, metyrapone was stopped and cortisol levels were monitored. It was noted that her cortisol levels were consistently below 250 $\mathrm{nmol} / \mathrm{L}$ with a corresponding ACTH of $34 \mathrm{ng} / \mathrm{L}$. A midnight cortisol after discontinuation of metyrapone for 2 weeks was low at $41 \mathrm{nmol} / \mathrm{L}$ consistent with spontaneous resolution of Cushing's syndrome. An insulin tolerance test showed sub-optimal cortisol response and she was commenced on hydrocortisone replacement therapy. However, within 3 months since discharge, she represented to hospital with reduced mobility and hypokalaemia. A LDDST after stopping hydrocortisone, confirmed relapse of Cushing's syndrome with a cortisol of $>1650$ at 48 hours. IPSS excluded a central ACTH source (Table 1) and a gallium 68 DOTATATE PET CT identified a $1.6 \mathrm{~cm}$ gallium avid lung lesion consistent with possible ectopic source. (Figure 1 and 2)

TABLE 1: IPSS- Suggestive of ectopic ACTH source

\begin{tabular}{|l|l|l|l|}
\hline Time & Plasma ACTH (ng/l) & \multicolumn{2}{l|}{} \\
\hline (min) & Left petrosal sinus & Right petrosal sinus & Peripheral Vein \\
\hline $\mathbf{- 5}$ & 483 & 491 & 440 \\
\hline $\mathbf{0}$ & 449 & 451 & 423 \\
\hline $\mathbf{2}$ & 490 & 516 & 430 \\
\hline $\mathbf{5}$ & 488 & 527 & 401 \\
\hline $\mathbf{1 0}$ & 515 & 562 & 480 \\
\hline Time & Plasma Cortisol (nmol/I) & \multicolumn{2}{|l|}{} \\
\hline (min) & Left petrosal sinus & Right petrosal sinus & Peripheral Vein \\
\hline $\mathbf{- 5}$ & 2160 & 2277 & 2143 \\
\hline $\mathbf{0}$ & 2178 & 2151 & 2139 \\
\hline $\mathbf{2}$ & 2128 & 2072 & 2181 \\
\hline $\mathbf{5}$ & 2165 & 2107 & 2119 \\
\hline $\mathbf{1 0}$ & 2227 & 2222 & 2113 \\
\hline Time & Plasma Prolactin (mU/l) & \multicolumn{2}{|l|}{} \\
\hline $\mathbf{( m i n )}$ & Left petrosal sinus & Right petrosal sinus & Peripheral Vein \\
\hline $\mathbf{- 5}$ & 317 & 887 & 195 \\
\hline $\mathbf{0}$ & 284 & 685 & 177 \\
\hline $\mathbf{2}$ & 393 & 1185 & 176 \\
\hline $\mathbf{5}$ & 548 & 1029 & 175 \\
\hline $\mathbf{1 0}$ & 517 & 787 & 186 \\
\hline
\end{tabular}

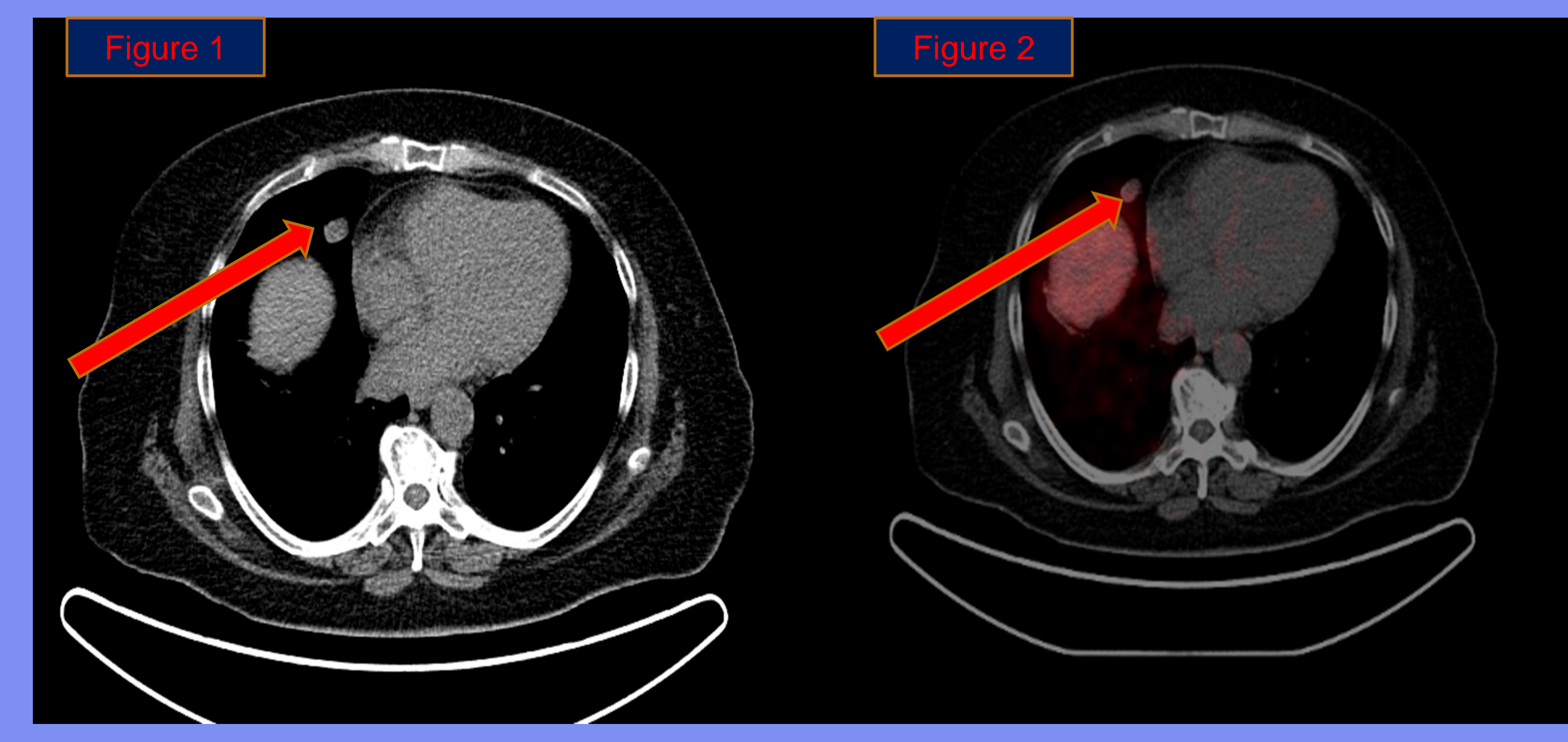

FIGURE 1: CT Chest- suggestive of $1.3 \mathrm{~cm}$ right lower lung lobe lesion

FIGURE 2: Gallium 68 DOTATATE PET CT confirming a gallium avid right lower lung lobe lesion

\section{Management}

Once relapse was confirmed, she was restarted on $500 \mathrm{mg}$ tds of metyrapone with a prompt reduction of ACTH to $46 \mathrm{ng} / \mathrm{l}$ with cortisol of $79 \mathrm{nmol} / \mathrm{L}$. She underwent a right middle lobectomy 8 months after her initial presentation (Nov 2013). This was an uncomplicated operation and she was discharged on hydrocortisone replacement therapy. Histology of the resected lung lesion confirmed a well differentiated neuroendocrine tumour with no lymphovascular invasion and $\mathrm{Ki}-67$ of $<3 \%$. Features were typical of carcinoid pT1a; however changes consistent with diffuse idiopathic pulmonary neuroendocrine hyperplasia (DIPNECH) were also found.

FIGURE 3: Course of ACTH and cortisol over 8 months

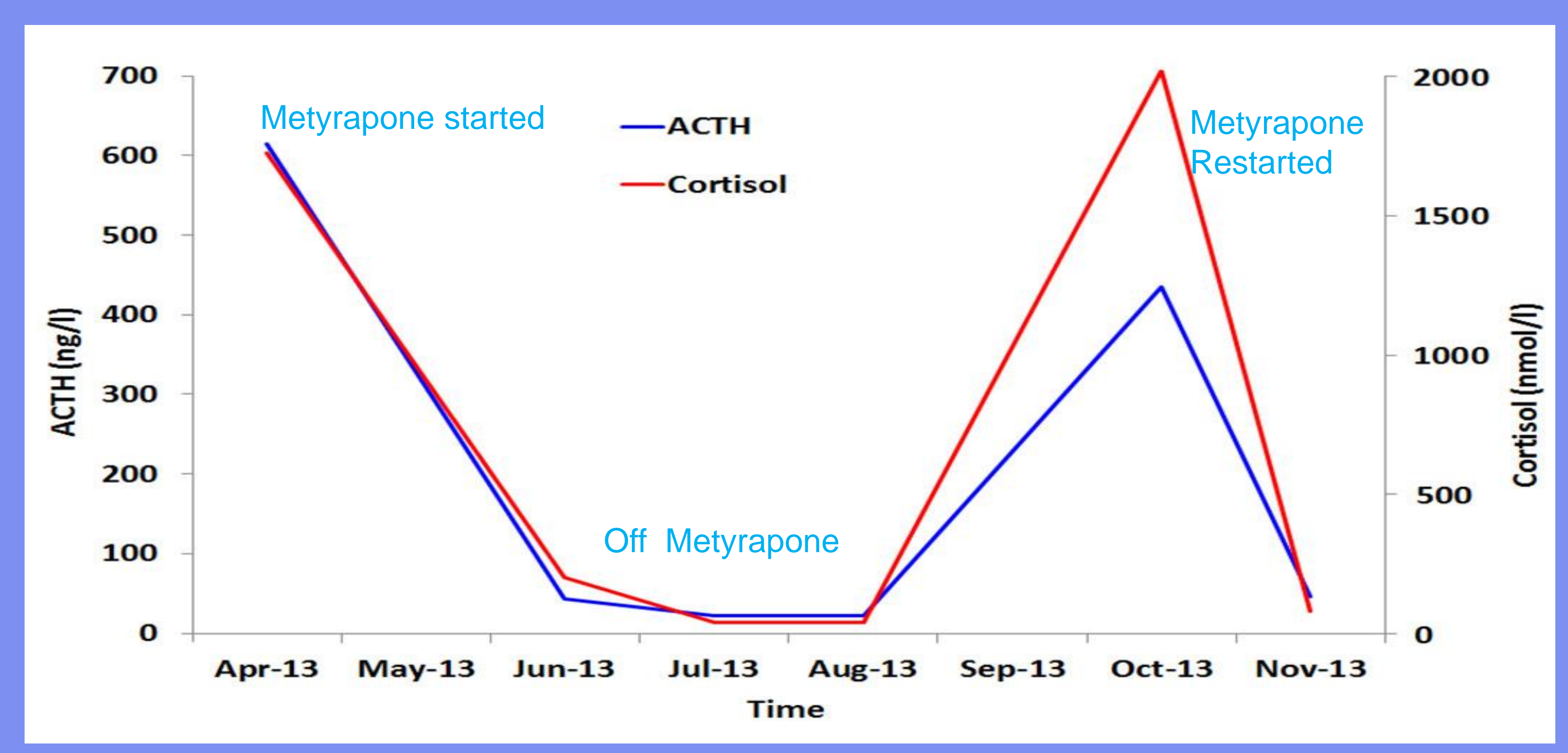

\section{Learning points}

- In patients with Cushing's syndrome whose cortisol levels respond briskly to low dose metyrapone, spontaneous remission should be considered

- Careful monitoring of patients with Cushing's, who appear to recover spontaneously is essential as relapses are frequent

- This is a very unusual case as although cyclical Cushing's is well recognised in Cushing's disease, it is very rare with ectopic ACTH production

- To the best of our knowledge, this is only the second reported case of DIPNECH associated with cyclical ectopic Cushing's. 